\title{
The Raman spectra of four isotopic varieties of diborane in the gas phase
}

\author{
R. C. TAYLor and A. R. EMERY \\ Department of Chemistry, University of Michigan, Ann Arbor, Michigan
}

(Received 13 September 1957)

\begin{abstract}
Raman spectra of the four isotopic species, ${ }^{11} \mathrm{~B}_{2} \mathrm{H}_{6},{ }^{11} \mathrm{~B}_{2} \mathrm{D}_{6},{ }^{10} \mathrm{~B}_{2} \mathrm{H}_{6},{ }^{10} \mathrm{~B}_{2} \mathrm{D}_{6}$, in the gas phase have been obtained and interpreted in terms of the $D_{2 h}$ bridge model. The results are in agreement with previous interpretations in the literature based on the spectrum of the liquid.
\end{abstract}

THE compounds ${ }^{11} \mathrm{~B}_{2} \mathrm{H}_{6},{ }^{11} \mathrm{~B}_{2} \mathrm{D}_{6},{ }^{10} \mathrm{~B}_{2} \mathrm{H}_{6}$ and ${ }^{10} \mathrm{~B}_{2} \mathrm{D}_{6}$ were prepared during the course of a spectroscopic investigation of the borohydride ion and aluminum borohydride. Since the Raman spectra of only the first and last of the above isotopic varieties have been reported in detail, and those only for the liquid state, it was deemed of interest to obtain the gaseous Raman spectra for the complete series.

\section{Experimental}

The starting material in the preparation of the compounds was the diethyl ether complex of $\mathrm{BF}_{3}$. This was converted to the appropriate diborane by means of lithium aluminum hydride or deuteride essentially according to the method given by Sraprro et al. [1]. The boron-11 compounds contained approximately 81 per cent of the ${ }^{11 B}$ isotope, the normal isotopic abundance, while the boron-10 compounds* contained 96 per cent of the desired isotope. The hydrogen content of the deuterated compounds was estimated at less than 2 per cent from the spectral evidence. Purification was accomplished by repeated fractionation on the vacuum line until a constant and reproducible vapor pressure was reached. The values observed for ordinary diborane agreed with those in the literature. The use of $\mathrm{BF}_{3}$ etherate as a starting muterial eliminates possible contamination with $\mathrm{SiF}_{4}$, which is difficult to remove by fractionation.

The spectra were recorded photographically, using the spectrograph and light source des. cribed previously [2]. The Raman tubes had an outside diameter of $25 \mathrm{~mm}$ with an illuminated length of $150 \mathrm{~mm}$ and contained the gases at approximately 4 atmospheres pressure. Collimating baffles largely eliminated light scattered from the walls and window. Saturated sodium nitrite solution served as a filter for all exposures; an isopropyl alcohol solution of rhodamine $5 \mathrm{GDN}$ Extra and paranitrotoluene was utilized as an additional filter for a few of the spectra. Exposure times varied from 12 to $72 \mathrm{hr}$ with Eastman $103 \mathrm{a}-\mathrm{J}$ plates. The resolution of the spectrograph was quite adequate to resolve clearly the isotopie triplet of ordinary diborane at about $800 \mathrm{~cm}^{-1}$. Wave length measurements were made both on the plates and on enlarged tracings made by a Leeds and Northrup microphotometer. Estimated probable errors accompany the averaged values listed for the frequencies.

\section{Results}

Observed frequencies, estimated intensities, and assignments for the hydrogen and deuterium compounds, respectively, are shown in Tables 1 and 2. Tracings of typical spectra of the deuterated compounds are shown in Fig. 1. No difficulty was met in identifying the $A_{g}$ fundamentals, since the characteristic sharp appearance of totally symmetric bands in the Raman spectrum of gaseous compounds is at

* Boron-10 was obtained as the $\mathrm{CaF}_{2} \cdot \mathrm{BF}_{3}$ complex from Union Carbide and Carbon, Oak Ridge National Laboratory. 
R. C. Taylor and A. R. Emery

Table 1. Observed Raman frequencies of gaseous ${ }^{11} \mathrm{~B}_{2} \mathrm{H}_{6}$ and ${ }^{10} \mathrm{~B}_{2} \mathrm{H}_{6}$

\begin{tabular}{|c|c|c|c|c|c|c|c|}
\hline \multicolumn{2}{|c|}{${ }^{11} \mathrm{~B}_{2} \mathrm{H}_{6}$} & \multicolumn{2}{|c|}{${ }^{10} \mathrm{~B}_{2} \mathrm{H}_{6}$} & \multirow{2}{*}{ Intensity } & \multirow{2}{*}{ Shape } & \multirow{2}{*}{ Symmetry } & \multirow{2}{*}{ Assignment } \\
\hline $\begin{array}{c}\text { Frequency } \\
\left(\mathrm{cm}^{-1}\right)\end{array}$ & $\pm \delta$ & $\begin{array}{l}\text { Frequency } \\
\left(\mathrm{cm}^{-1}\right)\end{array}$ & $\pm \delta$ & & & & \\
\hline - & - & 683 & 3 & $v w$ & $s h$ & $A_{g}$ & $v_{y}-v_{10}$ \\
\hline 一 & - & 786 & 2 & $v w$ & $b r$ & & \\
\hline 788 & 1 & - & - & $s$ & $s h$ & $A_{g}$ & $v_{4}\left({ }^{11} \mathrm{~B}-{ }^{11} \mathrm{~B}\right)$ \\
\hline 802 & 1 & - & - & $m$ & $s h$ & $A_{g}^{g}$ & $v_{4}\left({ }^{11} \mathrm{~B}-{ }^{10} \mathrm{~B}\right)$ \\
\hline 818 & 2 & 816 & 1 & $s$ & $s h$ & $A_{g}$ & $v_{4}\left({ }^{10} \mathrm{~B}-{ }^{10} \mathrm{~B}\right)$ \\
\hline - & - & 832 & 3 & vow & $s h$ & & \\
\hline 1026 & 6 & 1025 & 5 & $w$ & $b r$ & & $\nu_{7} ?, v_{15} ?$ \\
\hline 1184 & 1 & 1186 & I & $m$ & $s h$ & $A_{g}$ & \\
\hline 1310 & 5 & 1318 & 2 & $w-m w$ & $s h$ & $A_{g}$ & $v_{9}+v_{10}$ \\
\hline 1755 & 5 & 1768 & , 8 & $w$ & $b r$ & $B_{1 g}$ & $v_{6}$ \\
\hline 2011 & 2 & - & 一 & vw & $s h$ & $A_{g}$ & $2 v_{15} ?$ \\
\hline 2109 & 1 & 2110 & 1 & $s$ & $s h$ & $A_{g}$ & $v_{2}$ \\
\hline- & - & 2352 & 2 & $w$ & $s h$ & $A_{g}$ & $2 v_{3}, 2 v_{18}$ \\
\hline 2485 & 7 & - & 一 & $v v w$ & & $A_{g}$ & $v_{4}+2 v_{5} ?$ \\
\hline 2532 & 1 & 2537 & 1 & $v s$ & $s h$ & $A_{g}$ & $\nu_{1}$ \\
\hline$(2600)$ & 10 & 2640 & 10 & $w$ & $b r$ & $B_{2 g}$ & $v_{11}$ \\
\hline
\end{tabular}

$\delta=$ probable error; $s=$ strong, $m=$ medium, $w=$ weak, $s h=$ sharp, $b r=$ broad; frequencies enclosed in parentheses are estimated; assignments followed by a question mark are based on fundamental frequencies obtained indirectly [3]

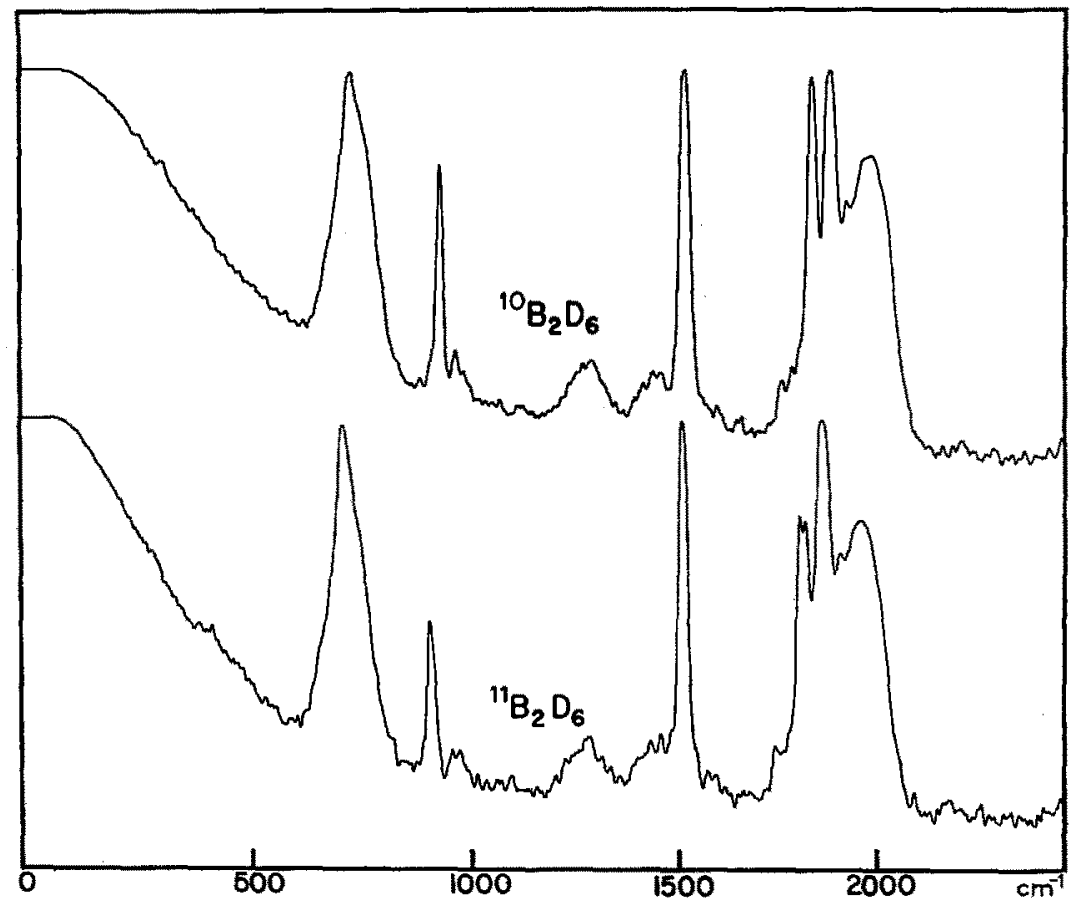

Fig. 1. Raman spectra of ${ }^{10} \mathrm{~B}$ and ${ }^{11} \mathrm{~B}$ deuteroboranes in the gas phase. 
The Raman spectra of four isotopic varieties of diborane in the gas phase

Table 2. Observed Raman frequencies of gaseous ${ }^{11} B_{2} D_{6}$ and ${ }^{10} B_{2} D_{6}$

\begin{tabular}{|c|c|c|c|c|c|c|c|}
\hline \multicolumn{2}{|c|}{${ }^{11} B_{2} D_{6}$} & \multicolumn{2}{|c|}{${ }^{10} \mathrm{~B}_{2} \mathrm{D}_{6}$} & \multirow{2}{*}{ Intensity } & \multirow{2}{*}{ Shape } & \multirow{2}{*}{ Symmetry } & \multirow{2}{*}{ Assignment } \\
\hline $\begin{array}{l}\text { Frequency } \\
\left(\mathrm{cm}^{-1}\right)\end{array}$ & $\pm \delta$ & $\begin{array}{l}\text { Frequency } \\
\left(\mathrm{cm}^{-1}\right)\end{array}$ & $\pm \delta$ & & & & \\
\hline 712 & 1 & 721 & 1 & $s$ & $s h$ & $A_{g}$ & $v_{4}$ \\
\hline - & - & 748 & 5 & $w$ & $b r$ & & $v_{7}, v_{12}, v_{15} ?$ \\
\hline 912 & 1 & 928 & 1 & $m$ & $s h$ & $A_{g}$ & \\
\hline 971 & 3 & 962 & 3 & $v w$ & $s h$ & $A_{g}$ & $v_{16}-v_{18} ?$ \\
\hline 1287 & 4 & 1285 & 5 & $w$ & $b r$ & $B_{1 g}^{g}$ & \\
\hline 1414 & 3 & 1446 & 3 & $v w$ & $s h$ & $A_{g}$ & \\
\hline 1438 & 3 & - & - & $v w$ & sh & $A_{g}^{g}$ & $2 v_{14}$ \\
\hline 1459 & 3 & - & - & $v w$ & $s h$ & $A_{g}$ & $2 v_{7}, 2 v_{12} ?$ \\
\hline 1488 & 4 & - & - & $v w$ & $s h$ & $A_{g}$ & $2 y_{1}+2$ \\
\hline 1515 & 1 & 1515 & 1 & $v 8$ & $s h$ & $A_{g}$ & $v_{2}$ \\
\hline 1748 & 2 & 1758 & 3 & $w w$ & $s h$ & $A_{g}$ & $2 v_{18}$ \\
\hline 1809 & 1 & 1836 & 1 & $s$ & $s h$ & $A_{g}$ & $2 y_{3}$ \\
\hline 1824 & 1 & - & 一 & $m$ & $s h$ & $A_{g}$ & $2 v_{3}\left({ }^{11} \mathrm{~B}-{ }^{10} \mathrm{~B}\right)$ \\
\hline 1867 & 1 & 1883 & 1 & vs & $s h$ & $A_{g}$ & $\nu_{1}$ \\
\hline 1912 & 2 & 1924 & 2 & $w$ & $s h$ & $A_{g}$ & $v_{4}+2 v_{5}$ \\
\hline 1968 & 2 & 1990 & 2 & $m$ & $b r$ & $B_{2 g}$ & $v_{11}$ \\
\hline
\end{tabular}

$\delta=$ estimated probable error; $s=$ strong, $m=$ medium, $w=$ weak, $s h=$ sharp, $b r=$ broad; assignments accompanied by a question mark are based on fundamental frequencies obtained indirectly [3].

least as conclusive as depolarization measurements. The other Raman-active fundamentals were typically broad and diffuse and lower in intensity so that frequently they could not be distinguished from the background. In some cases, they were obscured by $A_{g}$ fundamentals or overtones. This appears to be the reason for the great breadth of the band near $700 \mathrm{~cm}^{-1}$ in the deuterated compounds.

Assignment of overtones and combination bands was made with the help of the infrared active fundamentals of the gas listed by LORD and NIELSEN [3]; where Raman-active fundamentals could not be identified from the present work, liquid values from the same paper were used. Frequently alternate possibilities existed for the assignment of weak bands. In such cases, the assignment with $A_{g}$ symmetry, if one existed, was preferred on the grounds that bands of this class would likely be more intense in the Raman effect. Several cases in which the intensity of the overtone or combination was enhanced by Fermi resonance were noted, the most pronounced case occurring in the deuterated compounds and involving $2 v_{3}$ and $\nu_{1}$. The doublet appearing at the position of $2 \nu_{3}$ in the spectrum of ${ }^{11} \mathrm{~B}_{2} \mathrm{D}_{6}$ is attributed to the presence of roughly 30 per cent of ${ }^{11} \mathrm{~B}-{ }^{10} \mathrm{~B}$ molecules. The unexpectedly high intensity of the isotopic satellite at $1824 \mathrm{~cm}^{-1}$ above that expected from the ratio of isotopic molecules arises from a closer resonance with the $v_{1}$ fundamental in the lighter molecules.

The fundamentals derived from the present work are collected in Table 3. Since they agree quite well with those given by LORD and NIELSEN [3], who have considered all previous work, they will not be disoussed in detail. It may be 
pointed out that the general agreement found here supports the essential correctness of the assignments made by these authors.

The application of the Teller-Redlich product rule and also one of the isotopic sum rules to the frequencies of the $A_{g}$ class is shown in Table 4 . The data are satisfactory considering the lack of knowledge of the anharmonicities and the number of fundamentals involved in Fermi resonances.

Table 3. Raman active fundamentals of gaseous diboranes

\begin{tabular}{|c|c|c|c|c|c|}
\hline & & ${ }^{1{ }^{1} \mathrm{~B}_{2}} \mathrm{H}_{6}$ & ${ }^{10} \mathrm{~B}_{2} \mathrm{H}_{8}$ & ${ }^{11} \mathrm{~B}_{2} \mathrm{D}_{6}$ & ${ }^{10} \mathrm{~B}_{2} \mathrm{D}_{6}$ \\
\hline \multirow[t]{4}{*}{$A_{g}$} & $v_{1}$ & 2532 & 2537 & $(1860)$ & $(\mathbf{1 8 7 0})$ \\
\hline & $v_{2}$ & 2109 & 2110 & (1512) & (1514) \\
\hline & $y_{3}$ & 1184 & 1186 & $(912)$ & $(928)$ \\
\hline & $v_{4}$ & 788 & 816 & 712 & 721 \\
\hline \multirow[t]{2}{*}{$B_{1 g}$} & $v_{6}$ & 1755 & 1768 & 1287 & 1285 \\
\hline & $v_{7}$ & $-m$ & - & 一 & $=$ \\
\hline \multirow[t]{2}{*}{$B_{2 g}$} & $v_{11}$ & 2600 & 2640 & 1968 & 1990 \\
\hline & $v_{12}$ & - & - & 一 & - \\
\hline$B_{3 g}$ & $v_{15}$ & - & - & 一 & - \\
\hline
\end{tabular}

Frequencies enclosed in parentheses are involved in Fermi resonances and have been corrected by an estimated shifi.

Table 4. Product and sum rules for the $A_{g}$ class frequencies of gaseous diboranes

\begin{tabular}{l|l|l}
\hline Produet ratio & Calc. & Theoret. \\
\hline & & \\
$\left({ }^{11} \mathrm{~B}_{2} \mathrm{H}_{6} / 1 \mathrm{~B}_{2} \mathrm{D}_{6}\right)$ & $2 \cdot 73$ & $2 \cdot 825$ \\
$\left({ }^{10} \mathrm{~B}_{2} \mathrm{H}_{6} /{ }^{10} \mathrm{~B}_{2} \mathrm{D}_{6}\right)$ & $2 \cdot 74$ & $2 \cdot 825$ \\
$\left({ }^{10} \mathrm{~B}_{2} \mathrm{H}_{6} /{ }^{11} \mathrm{~B}_{2} \mathrm{H}_{6}\right)$ & 1.04 & $1 \cdot 049$ \\
$\left({ }^{10} \mathrm{~B}_{2} \mathrm{D}_{6} /{ }^{11} \mathrm{~B}_{2} \mathrm{D}_{6}\right)$ & 1.04 & 1.049
\end{tabular}

Sum difference $\Sigma\left[v_{i}^{2}\left({ }^{10} \mathrm{~B}_{2} \mathrm{H}_{6}\right)-v_{2}{ }^{2}\left({ }^{11} \mathrm{~B}_{2} \mathrm{H}_{6}\right)\right]=7.9 \times 10^{4}$

$$
\Sigma\left[v_{i}^{2}\left({ }^{10} B_{2} D_{6}\right)-v_{2}^{2}\left({ }^{11} B_{2} D_{6}\right)\right]=8.6 \times 10^{4}
$$

Acknowledgements - This work was supported in part by the United States Air Force under Contract AF 33(616)-3343, the sponsoring agency being the Aeronautical Research Laboratory of the Wright Air Development Center, Air Research and Development Command.

\section{References}

[1] Shaptro T., Weiss H. G., Schmich M., Skolnik S. and Smith G. B. L. J. Amer. Chem. Soc. 195274901 .

[2] Vroale G. L. and TAYLor R. C. J. Amer. Chem. Soc. 195678294.

[3] Lond R. C. and NIELSEN E. J. Chem. Phys. 1951191. 